\title{
Pulse Shape Discrimination Properties of Plastic Scintillators Incorporating a Rationally Designed Highly Soluble and Polymerizable Derivative of 9,10-Diphenylanthracene
}

\author{
Tibor Jacob Hajagos, David Kishpaugh, Qibing Pei* \\ UCLA Department of Materials Science $\mathcal{E}$ Engineering, 420 Westwood Plaza, Los Angeles CA 90095, USA
}

\begin{abstract}
A highly soluble and polymerizable derivative of 9,10-diphenylanthracene was designed and synthesized specifically to be capable of achieving very high loadings (at least 50 wt.\%) when copolymerized with a polyvinyltoluene (PVT) matrix. The resulting heavily crosslinked plastics are mechanically hard and robust, and were found to have exceptional clarity with no sign of dye precipitation. Samples of these plastics both with and without added wavelength shifter were characterized for light yield, scintillation decay, and pulse shape discrimination (PSD) performance for $\alpha / \gamma$ discrimination, and the results were compared to that of a commercially available PSD plastic, EJ-299-34. The best performing formulation, with a primary dye loading of $50 \mathrm{wt} . \%$, had a measured light yield of 9950 photons/MeV, and achieved a PSD figure-of-merit (FOM) of 1.05 , the latter indicating that while the present material is not suited for practical applications, the overall approach demonstrates a proof-of-concept of PSD in highly loaded plastics stabilized through copolymerization of the primary dye, and suggests that further improvements through better dye choice/design may yet be achievable.
\end{abstract}

Keywords: Plastic Scintillator, Pulse Shape Discrimination (PSD), 9,10-Diphenylanthracene, Polymerizable Dye

\footnotetext{
${ }^{*}$ Corresponding Author. Tel.: +13108254217, Fax: +13102067353

Email addresses: tiborhaj@ucla.edu (Tibor Jacob Hajagos), dkishpaugh@ucla.edu (David Kishpaugh), qpei@seas.ucla.edu (Qibing Pei)
}

Preprint submitted to Nuclear Instruments and Methods A

March 28, 2016

(C) 2016. This manuscript version is made available under the Elsevier user license http://www.elsevier.com/open-access/userlicense/1.0/ 


\section{Introduction}

The variation in pulse shape originating from excitation from different high-energy particles is the origin of the technique of pulse shape discrimination (PSD), a technique that can be used to actively identify the type of the detected particle. In contrast to inorganic scintillators, which are among the best materials for $\gamma$-ray detection and spectroscopy, organic materials are among the most widely used materials for PSD applications [1], a primary example of which is the use of PSD based detection methods for fast- $\mathrm{n} / \Upsilon$ discrimination, which is of particular interest for the detection of special nuclear material (SNM). The PSD properties of organic scintillators have a long history dating back to the 1950's [2]. Organic crystals are among the highest performing materials for $\mathrm{n} / \curlyvee$ PSD, with single crystal trans-stilbene being readily considered as having the best known PSD performance [2,3]. On the other hand, PSD is readily achievable in conventional liquid scintillator solutions, which have distinct advantage over organic crystals in terms of cost, scalability, and ease of manufacture and handling. There are some distinct disadvantages of organic liquids, including concerns over toxicity, flammability, the potential for leaks, and the necessity of maintaining strict oxygen free conditions of the solution throughout its life, all of which are even more problematic in the context of very large detector sizes. Despite these shortcomings, liquid scintillator solutions are among the most widely used detector materials for fast neutron spectroscopy and $\mathrm{n} / \Upsilon$ discrimination.

For other types of detection, plastic scintillators offer many distinct advantages over organic or even inorganic crystals and liquid scintillator solutions, most notably low cost, very good scalability, and great ease of use and handling, with complex geometries being readily attainable due to the good machinability of typical plastic formulations. Despite all these advantages, plastic materials have until only recently long been regarded as unsuitable for PSD applications due to their inferior discrimination properties. A notable exception to this, however, is the development by Brooks et al. in 1960 of a PSD capable plastic scintillator dubbed as "Plastic 77" by the authors [4], whose commercialization was attempted under the trade name NE-150 but which was eventu- 
ally abandoned due to issues with the material's long-term stability [5]. Only in last few years has the demonstration of commercial plastic scintillators with efficient PSD properties been fully realized. The recent breakthrough was made in 2012 when Zaitseva et al. first reported the use of polyvinyltoluene (PVT) based scintillators with a high loading ( $\geq 30 \%)$ of 2,5-diphenyloxazole (PPO) as the first plastic scintillator since Brooks' that was capable of achieving efficient $n / \gamma$ discrimination [6]. The necessity for a high primary dye loading in plastic scintillators was hinted at in their previous work, which established the requirement in PSD capable mixed crystals of a high concentration of primary solute states in order to facilitate triplet-triplet annihilation leading to emission of delayed fluorescence [7]. Since then, the material has been developed into two different commercial materials from Eljen Technology, EJ-299-33A, which has better performance but is relatively soft for a scintillating plastic, and EJ-299-34, which has better mechanical properties but decreased discrimination performance [8]. As an example of the difference in their mechanical properties, in our hands, EJ-299-33A was found to be very difficult to machine using our protocol for fabricating small disks (typically $\varnothing 10 \mathrm{~mm} \times 2-5 \mathrm{~mm}$ ) from regular sheet stock, which we developed initially for conventional plastic scintillator formulations (e.g., EJ-212). While EJ-299-34 was much more easily machined via the same process, some additional care was needed to achieve good finish prior to polishing, and some additional complications were encountered when attempting to affix the samples for polishing with low melting point adhesives $\left(T_{M} \sim 55^{\circ} \mathrm{C}\right.$, actual working temp. $\left.\sim 60-80^{\circ} \mathrm{C}\right)$, namely some deformation and surface hazing. While alternative and more optimized machining techniques quite probably can achieve good results with either material, it would be advantageous to have a PSD capable plastic that in general has as good if not better machinability than traditional scintillating plastic formulations so that costs associated with the processing of the material can be minimized. The mechanical properties of the all these materials are directly related to the high dye loading that enables them to be efficient at pulse shape discrimination. With such high loadings, the dye molecules act as effective plasticizing agents, rendering the resulting material softer than either the base plastic alone or conventional plastic scintillator formulations. Martinez et al. have shown that substitution of of the aromatic polymer matrices with a non-aromatic material such as 
poly(methyl methacrylate) (PMMA) can be advantageous due to its higher hardness, but the discrimination performance was again diminished [9].

Another potential problem arising from the use of high primary dye loading is the long term stability of the material, which is a principal issue needing to be addressed with regards to the current generation of PSD capable plastics. The failure of Brook's "Plastic 77" to be fully realized as the NE-150 commercial material was largely due to the dramatic loss of transparency and whitening which took place over time [5], presumably due to precipitation of the highly loaded dye components. Blanc et al. have experimented further with the Plastic 77 composition and demonstrated that both incorporating polymerizable additives and cross-linking of the polymer matrix were effective means of preventing precipitation of the dye components [10]. In general, with no other means of fundamentally preventing dye crystallization, a high dye loading will quite likely lead to precipitation, either during fabrication or upon aging, since it will be quite likely that the dye will be above its solubility limit in the cured plastic. The reason for this is at the elevated polymerization temperature with which plastics are typically cured, the dye will typically have a significantly higher solubility than in the monomer at room temperature. If a plastic is cured with a dye content at or near the high temperature solubility limit, upon cooling to room temperature there will be a significant degree of supersaturation of the dye, which can lead to precipitation either during the cooling itself, leading to an opaque material, or slowly over time, leading to a gradual loss of transparency. Additionally, the thermodynamic solubility limit of the dye in a polymer may be fundamentally lower than in the liquid monomer at any given temperature, which again would lead to a situation of supersaturatiion of the dye in the cured plastic. Lower dye loadings can be utilized to improve long term stability of the material, but not without some sacrifice in PSD performance (at least for presently known PSD-capable dyes). As such, development of new approaches to stabilization of high dye loadings remains a key objective for the development of new PSD capable plastics.

In this work, we address several key factors to the design of novel primary dyes to specifically address all of the issues associated with the high dye loading method. First and foremost is the need for a primary dye with high solubility to achieve loadings 
capable of effecting good PSD performance, but without leading to precipitation of the dye or loss of mechanical properties, especially at timescales corresponding to the expected useful lifetime of the material. While PPO has been shown to be a PSD capable dye in plastic scintillators, it is anticipated that there are a number of other possible candidates, some of which may be able to achieve significantly better performance than even the best commercial PSD plastic. However, there are very few if any efficient and common scintillating dyes other than PPO or its derivatives that have solubilities in aromatic solvents high enough to achieve decent PSD performance [6, 11]. With all this in mind, our main approach was to identify a candidate scintillating dye with overall good properties and good PSD performance, and then functionally derivatize the parent molecule to overcome the shortcoming of solubility and stability in the final plastic. To achieve the former, the introduction of long, flexible, and/or branched alkyl chains can greatly enhance solubility while simultaneously lowering the compounds melting point, while for the latter, the introduction of polymerizable groups can allow for direct bonding of the dye to the polymer backbone, eliminating the ability of the dye to precipitate from the plastic upon cooling. It is worth noting that at least one previous attempt has been made, albeit unsucessfully, to directly incorporate a scintillating dye via coplymerization into a PSD-capable plastic. In their report, O'Bryan et al. demonstrated PMMA-based plastic scintillators loaded up to $30 \mathrm{wt} \%$ with a polymerizable trans-stilbene derivative, but the resulting material failed to demonstrate any performance for $\mathrm{n} / \gamma$ PSD [12].

With all of these factors in mind, we set out to synthesize an entirely new primary dye that was rationally designed to fulfill each of these key requirements. Our specific approach reported herein is based upon the derivatization of 9,10-diphenylanthracene (DPA), a scintillating dye known in single crystal form to posses both good PSD and overall scintillator performance [13-15], and fig. 1 details the chemical structure of our proposed primary dye (DPA-DHS, where DHS='dihexylstyrene'). Functionalization of the DPA core by introduction of aryl ether linkages onto the pendant phenyl rings introduces the flexible hexyl spacer groups, whose primary intended function is to enhance the solubility of the dye while simultaneously lowering its melting point, both of which are critical factors in allowing for the required high loading content. At the 


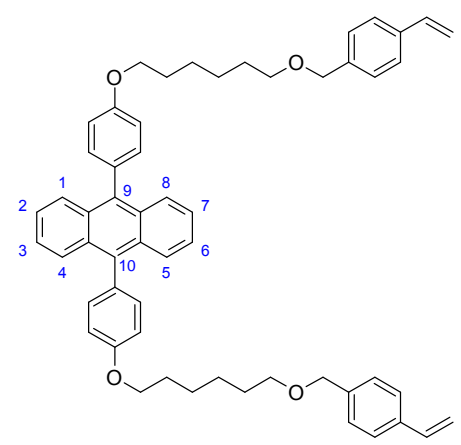

Figure 1: Structure of the DPA-DHS dye monomer (numbering of the anthracene core shown for reference).

distal end of the spacer chains are styrenyl groups, which are purposefully included in our proposed structure over other potential polymerizable groups in an attempt to achieve optimal incorporation of the dye into the overall polymer matrix. In addition, the presence of two polymerizable groups per dye molecule was intended to allow the dye to simultaneously act as a cross-linker for the matrix, and since high loadings are sought after, such a high degree of cross-linking would lead to a material that is quite hard and tough, potentially more so than the current generation of PSD plastics.

\section{Experimental}

\subsection{General Remarks}

All starting materials for synthetic procedures were purchased from commercial sources and used as received. Anhydrous THF was obtained via distillation over $\mathrm{Na}$ /benzophenone. Anhydrous DCM and DMF were purchased predried over molecular sieves. ${ }^{1} \mathrm{H}$ and ${ }^{13} \mathrm{C}$ NMR spectra were obtained using either a Bruker DRX500 spectrometer using a $5 \mathrm{~mm}$ broadband probe or a Bruker AV500 spectrometer with a 5 $\mathrm{mm}$ cryoprobe. Melting points were obtained using a microscope fitted with an Instec HC621V-F8 hot stage and mK1000 temperature controller.

\subsection{Overall Synthesis of the DPA-DHS Monomer}

The synthetic scheme for the synthesis of the DPA-DHS monomer is shown in fig. 2. There are five discreet steps in total with a four-step sequence being the longest 


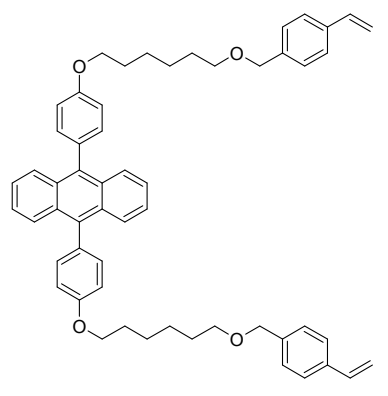

(DPA-DHS)

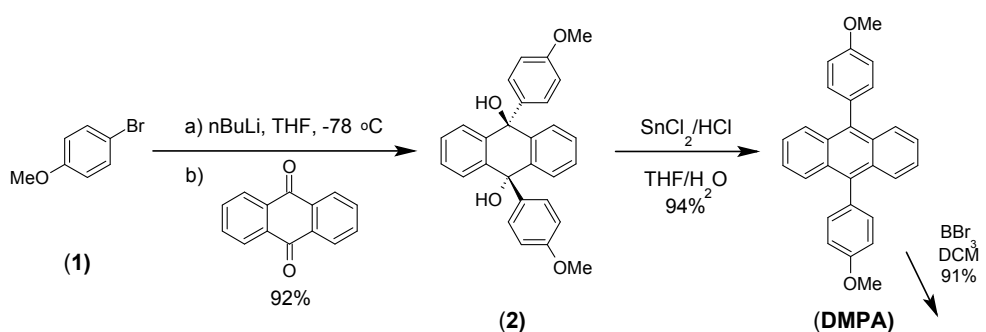

linear branch with an overall yield of 55\%. The synthesis includes as one of its intermediates the dimethoxy-functionalized DMPA, which was additionally utilized as a model compound to investigate the impacts to the photoluminescence properties of DPA-DHS by the introduction of the polymerizable side chains.

(2)

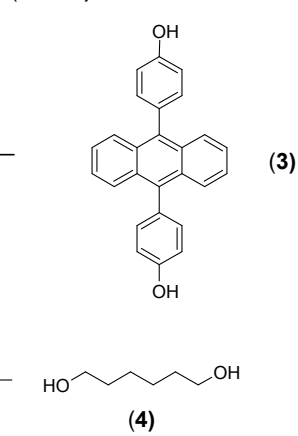

Figure 2: Synthetic Scheme for the synthesis of DPA-DHS.

\subsubsection{Synthesis of 9,10-Bis(4-methoxyphenyl)anthracene-9,10-diol (2)}

To a solution of $3.76 \mathrm{~mL}(30.0 \mathrm{mmol} ; 2.5$ eq.) of 4-bromoanisole (1) in $60 \mathrm{~mL}$ of dry THF at $-78{ }^{\circ} \mathrm{C}$ under Ar was dropwise added $12.0 \mathrm{~mL}$ (30.0 mmol; 2.5 eq.) of 2.5 $\mathrm{M}$ n-butyllithium in hexanes. After stirring at $-78^{\circ} \mathrm{C}$ for 1 hour, the solution was transferred dropwise via cannula to a suspension of $2.50 \mathrm{~g}(12.0 \mathrm{mmol} ; 1$ eq.) of anthraquinone in $50 \mathrm{~mL}$ of dry $\mathrm{THF}$ at $-78{ }^{\circ} \mathrm{C}$. The resulting solution was warmed to room temperature and stirred for 18.5 hours, then cooled to $0{ }^{\circ} \mathrm{C}$ and quenched with $20 \mathrm{~mL}$ of sat. $\mathrm{NH}_{4} \mathrm{Cl}$. The reaction contents were poured into $250 \mathrm{~mL}$ of $\mathrm{H}_{2} \mathrm{O}$, and the

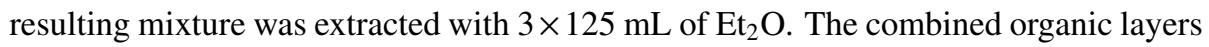
were washed with $2 \times 250 \mathrm{~mL}$ of sat. $\mathrm{NaCl}$ and dried over $\mathrm{MgSO}_{4}$. After removal of the solvent under vacuum, the crude product was preadsorbed onto silica gel before flash 
chromatography with 3:1 Hexane:EtOAc followed by 1:1 Hexane:EtOAc to give 4.69 $\mathrm{g}(92.0 \%$ yield $)$ of (2).

${ }^{1} \mathrm{H}-\mathrm{NMR}\left(500 \mathrm{MHz}, \mathrm{CDCl}_{3}\right) \delta=7.78-7.73(\mathrm{~m}, 4 \mathrm{H}), 7.40-7.35(\mathrm{~m}, 4 \mathrm{H}), 6.81-6.77(\mathrm{~m}$, 4H), 6.46-6.42 (m, 4H), $3.64(\mathrm{~s}, 6 \mathrm{H}), 2.97$ (s, 2H).

${ }^{13} \mathrm{C}-\mathrm{NMR}\left(500 \mathrm{MHz}, \mathrm{CDCl}_{3}\right) \delta=158.28,141.27,136.83,128.69,127.91,126.71$, $112.93,74.67,55.20$.

\subsubsection{Synthesis of 9,10-Bis(4-methoxyphenyl)-anthracene (DMPA)}

To a room temperature solution of $2.96 \mathrm{~g}(6.97 \mathrm{mmol}$; 1 eq. $)$ of (2) in $75 \mathrm{~mL}$ of THF that was sparged with Ar for 15 minutes was added a solution of $15.73 \mathrm{~g}$ (69.7 mmol; 10 eq.) of $\mathrm{SnCl}_{2} \cdot 2 \mathrm{H}_{2} \mathrm{O}$ in $60 \mathrm{~mL}$ of $10 \%(\mathrm{wt} / \mathrm{v}) \mathrm{HCl}$. The reaction was sparged with Ar for an additional 10 minutes, and after stirring at room temperature for 4.75 hours, the contents were diluted with $75 \mathrm{~mL}$ of $\mathrm{H}_{2} \mathrm{O}$ and filtered. The collected product was washed with $\mathrm{H}_{2} \mathrm{O}$ and $\mathrm{MeOH}$, dried under vacuum, and recrystallized from $\mathrm{CHCl}_{3} /$ cyclohexane to give $2.46 \mathrm{~g}$ (90.4\% yield) of (DMPA).

${ }^{1} \mathrm{H}-\mathrm{NMR}\left(500 \mathrm{MHz}, \mathrm{CDCl}_{3}\right) \delta=7.76-7.71(\mathrm{~m}, 4 \mathrm{H}), 7.42-7.37(\mathrm{~m}, 4 \mathrm{H}), 7.35-7.31(\mathrm{~m}$, $4 \mathrm{H}), 7.17-7.12(\mathrm{~m}, 4 \mathrm{H}), 4.00-3.94(\mathrm{~s}, 6 \mathrm{H})$.

${ }^{13} \mathrm{C}-\mathrm{NMR}\left(500 \mathrm{MHz}, \mathrm{CDCl}_{3}\right) \delta=159.15,136.89,132.53,131.29,130.40,127.18$, $125.03,114.00,55.55$.

\subsubsection{Synthesis of 9,10-Bis(4-hydroxyphenyl)-anthracene (3)}

To a suspension of $3.11 \mathrm{~g}$ (7.96 mmol; 1 eq.) of (DMPA) in $150 \mathrm{~mL}$ of dry DCM at $0{ }^{\circ} \mathrm{C}$ under Ar was dropwise added $17.5 \mathrm{~mL}$ (17.5 mmol; 2.2 eq.) of $1 \mathrm{M} \mathrm{BBr}_{3}$ in $\mathrm{DCM}$. The reaction was slowly warmed to room temperature and stirred for 16 hours, then cooled again to $0{ }^{\circ} \mathrm{C}$ before quenching with $30 \mathrm{~mL}$ of $10 \%(\mathrm{wt} / \mathrm{v}) \mathrm{HCl}$. The resulting mixture was warmed to room temperature, poured into $150 \mathrm{~mL}$ of $\mathrm{H}_{2} \mathrm{O}$, and extracted with $3 \times 175 \mathrm{~mL}$ of EtOAc. The combined organic layers were washed with $300 \mathrm{~mL}$ of $\mathrm{H}_{2} \mathrm{O}, 300 \mathrm{~mL}$ of sat. $\mathrm{NaHCO}_{3}$, and $300 \mathrm{~mL}$ of sat. $\mathrm{NaCl}$, then dried over $\mathrm{MgSO}_{4}$. After removal of the solvent under vacuum, the crude product was preadsorbed onto 
silica gel before flash chromatography with 2:1 Hexane:EtOAc to give $2.61 \mathrm{~g}(90.7 \%$ yield) of (3).

${ }^{1} \mathrm{H}-\mathrm{NMR}\left(500 \mathrm{MHz}, \mathrm{THF}-d_{8}\right) \delta=8.51(\mathrm{~s}, 2 \mathrm{H}), 7.75-7.70(\mathrm{~m}, 4 \mathrm{H}), 7.29-7.25(\mathrm{~m}, 4 \mathrm{H})$, 7.24-7.20 (m, 4H), 7.00-6.97 (m, 4H).

${ }^{13} \mathrm{C}-\mathrm{NMR}\left(500 \mathrm{MHz}, \mathrm{THF}-d_{8}\right) \delta=158.25,137.89,133.06,131.34,130.46,127.84$, $125.38,116.08$

\subsubsection{Synthesis of 6-[(4-Ethenylphenyl)methoxy]-1-hexanol (5)}

To a suspension of $2.00 \mathrm{~g}(50.0 \mathrm{mmol}$; 1 eq. $)$ of $60 \% \mathrm{NaH}$ in $100 \mathrm{~mL}$ of dry DMF at $0{ }^{\circ} \mathrm{C}$ under Ar was carefully added $17.73 \mathrm{~g}$ (150.0 mmol; 3 eq.) of 1,6-hexanediol (4) against a counterflow of Ar. The mixture was warmed to room temperature and stirred for 1 hour, then cooled to $0{ }^{\circ} \mathrm{C}$ before dropwise adding $7.75 \mathrm{~mL}(55.0 \mathrm{mmol}$; 1.1 eq.) of 4-vinylbenzyl chloride. The reaction was warmed to room temperature and stirred for 17.5 hours, then poured into $500 \mathrm{~mL}$ of $\mathrm{H}_{2} \mathrm{O}$ and extracted with $3 \times 200$ $\mathrm{mL}$ of $\mathrm{Et}_{2} \mathrm{O}$. The combined organic layers were washed with $2 \times 400 \mathrm{~mL}$ of $\mathrm{H}_{2} \mathrm{O}$ and $400 \mathrm{~mL}$ of sat. $\mathrm{NaCl}$, then dried over $\mathrm{MgSO}_{4}$. After removal of the solvent under vacuum, the crude product was purified via flash chromatography on silica gel with $3: 1$ Hexane:EtOAc to give $8.72 \mathrm{~g}$ (74.4\% yield) of (5).

${ }^{1} \mathrm{H}-\mathrm{NMR}\left(500 \mathrm{MHz}, \mathrm{CDCl}_{3}\right) \delta=7.39(\mathrm{~d}, J=8.0 \mathrm{~Hz}, 2 \mathrm{H}), 7.29(\mathrm{~d}, J=8.0 \mathrm{~Hz}$, $2 \mathrm{H}), 6.71(\mathrm{dd}, J=17.6,10.9 \mathrm{~Hz}, 1 \mathrm{H}), 5.74(\mathrm{dd}, J=17.6,0.9 \mathrm{~Hz}, 1 \mathrm{H}), 5.23(\mathrm{dd}$, $J=10.9,0.9 \mathrm{~Hz}, 1 \mathrm{H}), 4.48(\mathrm{~s}, 2 \mathrm{H}), 3.60(\mathrm{t}, J=6.6 \mathrm{~Hz}, 2 \mathrm{H}), 3.46(\mathrm{t}, J=6.6 \mathrm{~Hz}, 2 \mathrm{H})$, $1.82(\mathrm{~s}, 1 \mathrm{H}), 1.62$ (quin, $J=6.9 \mathrm{~Hz}, 2 \mathrm{H}), 1.56(\mathrm{t}, J=6.9 \mathrm{~Hz}, 2 \mathrm{H}), 1.43-1.32(\mathrm{~m}, 4 \mathrm{H})$.

${ }^{13} \mathrm{C}-\mathrm{NMR}\left(500 \mathrm{MHz}, \mathrm{CDCl}_{3}\right) \delta=138.29,136.95,136.64,127.92,126.28,113.77$, $72.66,70.37,62.86,32.74,29.77,26.07,25.66$.

\subsubsection{Synthesis of 9,10-Bis[4-(\{6-[(4-ethenylphenyl)methoxy]hexyl\}oxy)phenyl]anthra-} cene (DPA-DHS)

To a solution of $1.33 \mathrm{~g}$ ( $3.67 \mathrm{mmol} ; 1$ eq.) of (3), $2.58 \mathrm{~g} \mathrm{(11.0} \mathrm{mmol;} 3$ eq.) of (5), and $1.73 \mathrm{~mL}$ (11.0 mmol; 3 eq.) of diethylazodicarboxylate in $70 \mathrm{~mL}$ of dry THF at 
$0{ }^{\circ} \mathrm{C}$ under Ar was dropwise added a solution of $2.89 \mathrm{~g}$ (11.0 mmol; 3 eq. $)$ of triphenylphosphine in $25 \mathrm{~mL}$ of dry THF with the formation of a precipitate. The reaction was warmed to room temperature and stirred for 24 hours, after which all of the precipitate had redissolved. The reaction solvent was removed under vacuum, and the crude mixture was purified via flash chromatography on silica gel with 1:3 Hexane:DCM to give $2.39 \mathrm{~g}\left(81.9 \%\right.$ yield) of (DPA-DHS) as a pale yellow solid (m.p. 119-123 $\left.{ }^{\circ} \mathrm{C}\right)$.

${ }^{1} \mathrm{H}-\mathrm{NMR}\left(500 \mathrm{MHz}, \mathrm{CDCl}_{3}\right) \delta=7.78-7.73(\mathrm{~m}, 4 \mathrm{H}), 7.42-7.40(\mathrm{~m}, 4 \mathrm{H}), 7.39-7.36(\mathrm{~m}$, 4H), 7.36-7.31 (m, 8H), 7.15-7.11 (m, 4H), $6.72(\mathrm{dd}, J=17.6,10.9 \mathrm{~Hz}, 2 \mathrm{H}), 5.75(\mathrm{dd}$, $J=17.6,0.9 \mathrm{~Hz}, 2 \mathrm{H}), 5.23(\mathrm{dd}, J=10.9,0.9 \mathrm{~Hz}, 2 \mathrm{H}), 4.52(\mathrm{~s}, 4 \mathrm{H}), 4.11(\mathrm{t}, J=6.5$ $\mathrm{Hz}, 4 \mathrm{H}$ ), 3.52 (t, $J=6.5 \mathrm{~Hz}, 4 \mathrm{H}$ ), 1.91 (quin, $J=6.9 \mathrm{~Hz}, 4 \mathrm{H}$ ), 1.72 (quin, $J=6.9 \mathrm{~Hz}$, $4 \mathrm{H}), 1.63-1.49(\mathrm{~m}, 8 \mathrm{H})$. ${ }^{13} \mathrm{C}-\mathrm{NMR}\left(500 \mathrm{MHz}, \mathrm{CDCl}_{3}\right) \delta=158.45,138.20,136.80,136.70,136.48,132.26$, $130.85,130.17,127.76,126.96,126.14,124.74,114.28,113.61,72.55,70.21,67.88$, $29.67,29.28,25.99,25.95$.

\subsection{Scintillator Sample Fabrication}

Coumarin 6 (Sigma-Aldrich) and 1,1-di-(tert-butylperoxy)-3,3,5-trimethylcyclohexane (initiator; Acros) were used as received. Methystyrene (60\% meta, $40 \%$ para, $\& 1 \%$ ortho mixture of isomers; Sigma-Aldrich) was degassed via freeze-pump-thaw and passed through a short column of inhibitor remover (Sigma-Aldrich) prior to use. Glass shell vials with internal diameters of $10 \mathrm{~mm}$ were cleaned in piranha solution immediately before vapor treatment under house vacuum with (tridecafluoro-1,1,2,2tetrahydrooctyl)trichlorosilane (Gelest). All samples were prepared by mixing each component along with 0.5 wt. $\%$ of initiator and heating at $80{ }^{\circ} \mathrm{C}$ for 24 hours under $\mathrm{N}_{2}$. After cooling to room temperature, the vials were broken to remove the cured samples, which were further ground and polished to the desired thickness.

\subsection{Characterization Methods}

Absorbance spectra of solutions were acquired using a Shimadzu UV-1700 UVVisible Spectrophotometer and were referenced again a solvent blank. Photoluminescence spectra were acquired using a QuantaMaster QM-4/SE (Photon Technology 
Inc.) fluorometer. Emission spectra for solutions were acquired using $10 \mathrm{~mm}$ quartz cells in the standard right-angle geometry, while spectra for plastic samples were acquired using a right angle geometry specially offset to avoid collection of light emitted directly from the sample surface, in order to best represent the technical emission spectrum of the scintillator samples as viewed by a PMT during actual detection tests. Photoluminescence quantum yields $\left(\phi_{P L}\right)$ of solutions were measured via the absorbance and fluorescence spectra of a series of concentrations for degassed (freeze-pump-thaw) samples using DPA in cyclohexane $\left(\phi_{P L}=1\right)[16]$ as the reference standard, and the concentration of all solutions were kept sufficiently low as to achieve absorbances no greater than $0.1 \mathrm{AU}$ over the range of concentrations.

Gamma-ray spectra under ${ }^{137} \mathrm{Cs}$ irradiation were recorded using a Canberra Lynx Digital Signal Processor; a custom machined PTFE sample holder was used to ensure placement accuracy and optical grease was used to couple the sample to the window of a Hamamatsu R878 PMT, which itself was plugged into a Canberra Model 2007P Photomultiplier Tube Base/Preamplifier. All spectra were recorded using a trapezoidal shaping filter with a $1.0 \mu$ s rise time and a $0.5 \mu$ s flat-top time. The relative Compton edge positions were compared to identically sized specimens of EJ-212 (Eljen Technology, Inc.) that were machined and polished from larger pieces of sheet stock. The acquired photoluminescence data was used along with the quantum efficiency curve for the PMT photocathode to correct the relative Compton edge positions for the emission spectrum of each sample. In this manner, absolute light yields were obtained from the manufacturer's specification for the light yield of EJ-212 (10,000 photons/MeV).

Time resolved radioluminescence decay curves were measured using a time-correlated single photon counting (TCSPC) based approach utilizing a Hamamatsu H243150 assembly as the start PMT and a Hamamatsu H10721P-110 module fitted with an E5776-51 SMA fiber optic adapter as the stop PMT. A bare terminated $800 \mu \mathrm{m}$ multimode fiber was used along with a custom machined PTFE sample holder to allow the stop PMT to collect single photons from the sample; under ${ }^{137} \mathrm{Cs}$ irradiation, the sample was directly coupled using optical grease to the window of the start PMT, but under ${ }^{210}$ Po irradiation, the PTFE sample holder and source were placed inside a specially constructed vacuum chamber with a quartz disk end-window, onto which the 
start PMT was coupled. The signal from the stop PMT was fed into a Ortec Model 9326 Fast Preamplifier, and the resulting output along with the signal from the start PMT was digitized using a PicoScope Model 5244B Oscilloscope (Pico Technology, Inc.) operated in 8-bit mode with a digitization rate of $500 \mathrm{MS} / \mathrm{sec}$. The differences in arrival times between single photon pulses detected by the stop PMT and the main pulse detected by the start PMT were histogrammed to obtain the scintillation decay curves over a $2 \mu$ s interval.

Pulse-shape discrimination tests were performed under simultaneous $\alpha / \gamma$ irradiation, which was done in lieu of the more commonplace $n / \gamma$ PSD testing on account of the fact that NRC exempt quantities of both $\gamma$ and $\alpha$ (specifically ${ }^{210} \mathrm{Po}$ ) emitters are readily available, inexpensive, and can be purchased and handled without special precautions or license (no such NRC exempt quantity is currently available for neutron emitting sources). Pulses from samples under irradiation were acquired using a Hamamatsu R6321-100 PMT that was coupled to the quartz disk end-window of a specially constructed vacuum chamber. The sample under test was coupled to the vacuum side of the end-window using optical grease, and was held in place using a custom machined PTFE sample holder, which also held in place the mixed source assembled from separate ${ }^{210} \mathrm{Po}$ and ${ }^{137} \mathrm{Cs}$ sources. Raw pulses obtained from the PMT anode were digitized using the Picoscope Model 5244B Oscilloscope operated in 12-bit mode with a digitization rate of $500 \mathrm{MS} / \mathrm{sec}$ and were saved directly to disk for off-line analysis. Energy calibration of the resulting data sets was performed by histogramming the total integral of each pulse and finding the position of the Compton edge of the gamma distribution. Two-dimensional plots representing the PSD performance were obtained using a digital charge comparison method to give a plot of $\left(\mathrm{Q}_{\text {tail }} / \mathrm{Q}_{\text {total }}\right)$ versus equivalent particle energy (keV-ee) [17-21].

\section{Results \& Discussion}

\subsection{Spectral and Electronic Properties of the DPA-DHS Monomer}

The absorbance and photoluminescence spectra of DPA, DPA-DHS, and the intermediate compound DMPA, are shown in fig. 3 and fig. 4, respectively. In these 


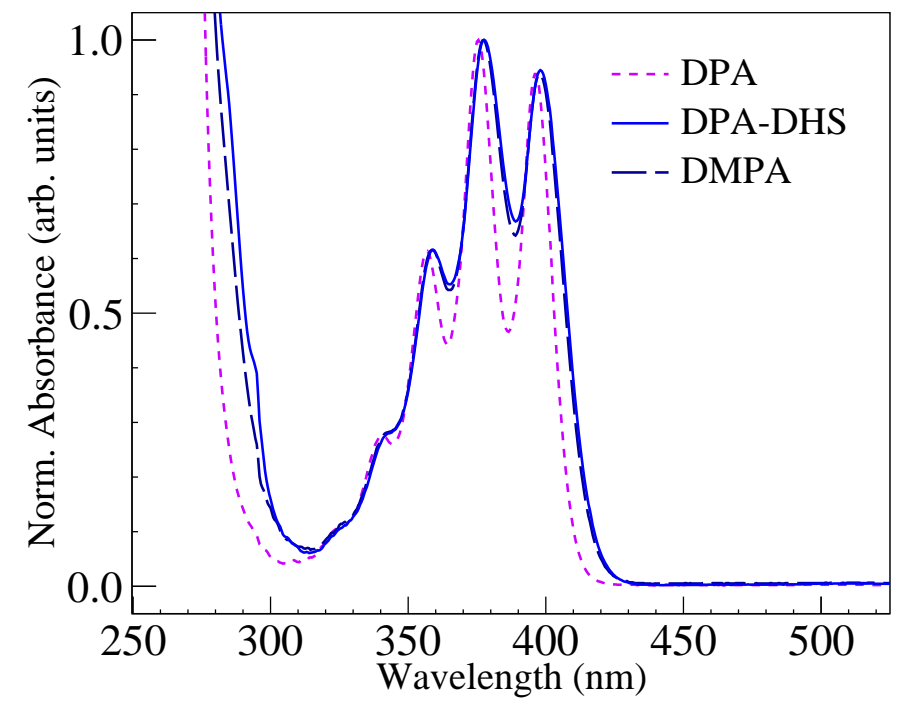

Figure 3: Absorbance spectra of DPA, DPA-DHS, and DMPA as dilute solutions in $\mathrm{CHCl}_{3}$.

spectra, the effects that the introduction of the ether linkage to the DPA core have on the electronic structure of DMPA and DPA-DHS can readily be observed. In fig. 3, a very slight red shifting $(\sim 2 \mathrm{~nm})$ of the absorbance maxima of DMPA and DPA-DHS is observed relative to DPA, and there is additional broadening of the vibronic sublevels for the former compounds. In fig. 4, the redshifting of the DMPA and DPA-DHS emission spectra relative to DPA is more prominent $(\sim 10 \mathrm{~nm})$, and again broadening of the vibronic sublevels is observable. Given that the value of the redshifting is much smaller for the absorbance spectrum, it is reasonable to conclude that the main effect of the introduction of the alkoxy substituents onto the electronic properties of DMPA and DPA-DHS is to introduce additional stabilization to their respective $S_{1}$ states relative to that of DPA, while leaving the transition energies at the corresponding ground state geometries relatively unchanged from one another. Additionally, the extra degrees of freedom to the vibrational modes introduced via the alkoxy substituents is a likely factor controlling the increased broadening of the vibronic sublevels of the spectra of DMPA and DPA-DHS. Overall, DMPA and DPA-DHS have virtually identical spectra to one another, which is indicative that increasing the length of the alkyl chain 


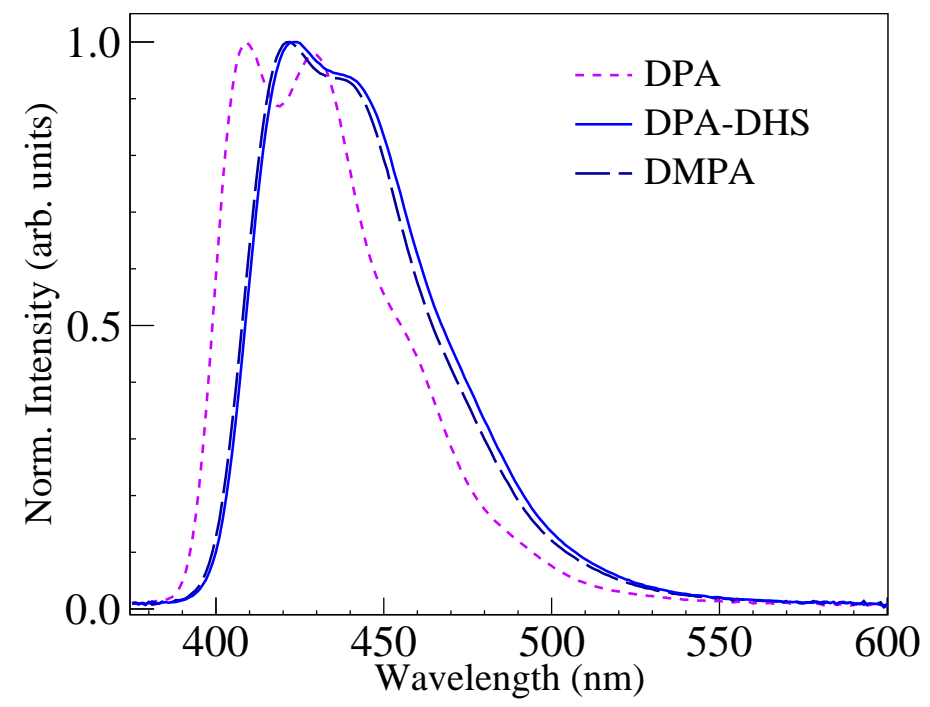

Figure 4: Photoluminescence Spectra of DPA, DPA-DHS, and DMPA as dilute solutions in toluene.

attached to the aryl ether linkage has little if any effect on the electronic properties of the DPA core. The photoluminescence quantum yields $\left(\phi_{P L}\right)$ of DMPA and DPA-DHS in toluene were measured to be 0.84 and 0.95 , respectively. The value of $\phi_{P L}$ measured for DMPA is noticeably higher than a previously reported value of 0.59 [22], although the authors do not specify whether the measurements were performed on deoxygenated solutions, which could account for the observed discrepancy. Overall, the modification of the DPA core to obtain the final structure of DPA-DHS, especially given the latter's high $\phi_{P L}$, does not appear to significantly disrupt its potential performance as a scintillating dye, at least in terms of its molecular emission.

\subsection{Fabrication $\mathcal{E}$ Photoluminescence of DPA-DHS/PVT Samples}

The DPA-DHS monomer obtained from our synthetic efforts was found to be quite amenable to processing through standard solution casting methods. A large reduction in melting point on account of the structural modifications was observed for DPA-DHS $\left(\sim 120^{\circ} \mathrm{C}\right)$ as compared to 9,10 -diphenylanthracene $\left(\sim 250^{\circ} \mathrm{C}\right)$. While loadings of $\leq 10$ wt. \% could dissolve in methylstyrene at room temperature, heating the undissolved mixture to the curing temperature of $80{ }^{\circ} \mathrm{C}$ along with gentle agitation was able to 
achieve full dissolution of loadings as high at $50 \mathrm{wt} . \%$. Higher loading may even be possible but become very difficult to achieve practically; as the DPA-DHS content is increased, the dye/monomer solution becomes very viscous, and with the high degree of cross-linking, the solution begins to rapidly gel within a few minutes, after which further mixing becomes impossible. The rapid gelation of the DPA-DHS/PVT samples (due to the cross-linking nature of its multiple polymerizable end-groups) is in direct contrast to DPA/PVT samples with DPA content ranging from 0.5-2.5 wt.\% fabricated for comparative purposes, for which noticeable gelation was observable only after a few hours into the curing.

The obtained plastic scintillator samples were found to good clarity, with no signs of phase separation, cloudiness, or precipitation of the primary dye. As a qualitative assessment of the outcome of the copolymerization, samples of DPA-DHS/PVT along with cast PVT obtained through the same polymerization procedure but with no added dye were subjected to swell tests in DCM. After several hours, which was an adequate amount of time to achieve full dissolution of the cast PVT, the DPA-DHS/PVT samples showed varying degrees of swelling, with the lowest loading of DPA-DHS tested (3 wt.\%) showing a significant volume expansion, and the highest loading (50 wt.\%) demonstrating only minimal volume expansion). Thus, the degree of crosslinking (i.e., resistance to swelling) is directly dependent on the loading of the DPA-DHS, which is a direct indicator of incorporation of the crosslinking DPA-DHS monomer into the polymer matrix (which is otherwise non-crosslinking). Additionally, some residual amount of unreacted or oligomeric DPA-DHS monomer was evidenced via the fluorescence of the swelling solution at the end of the tests, which is not unexpected given that residual monomer is commonly observed for the bulk polymerization of plastics [23, 24]. All of the DPA-DHS containing samples prepared were found to be mechanically robust and easily polished, as is to be expected from their crosslinking. DPA-DHS/PVT samples with no added wavelength shifter ranged from nearly colorless at low DPA-DHS content to a pale yellow in color at the highest DPA-DHS concentrations.

Additional DPA-DHS/PVT samples were prepared with 0.05 wt.\% of Coumarin 6 added to act as a wavelength shifter; Coumarin 6 was chosen for this role on account of the good matching of its absorbance spectrum to the molecular emission spectrum 
of DPA-DHS, which is illustrated in fig. 5. Wavelength shifters employed in most

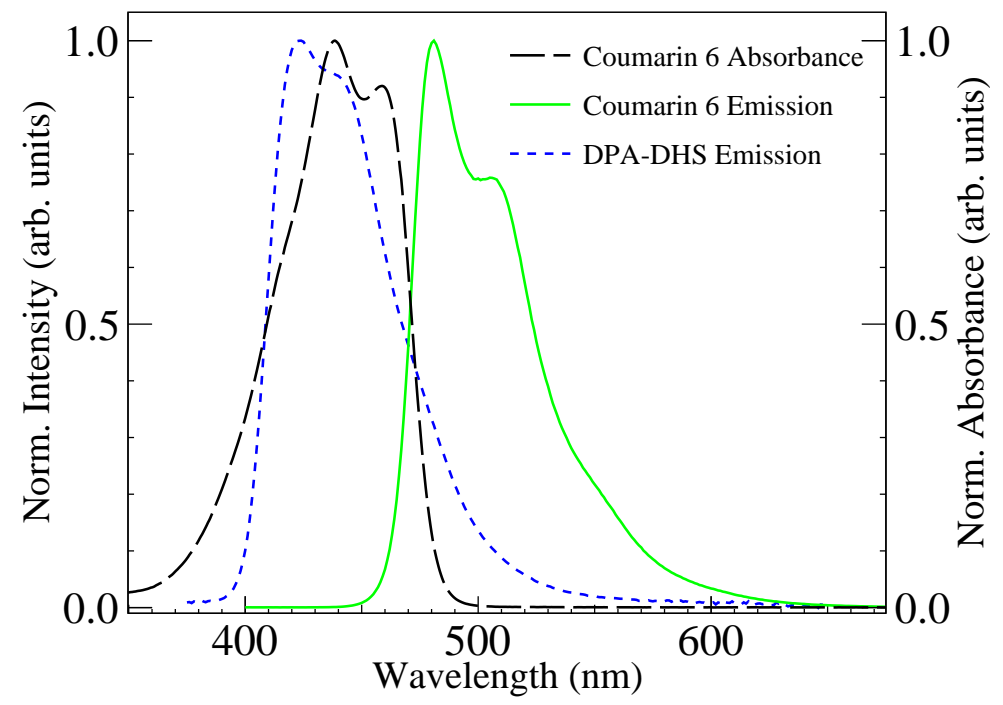

Figure 5: Emission/Absorbance spectra of Coumarin 6, overlaid with the emission spectrum of DPA-DHS, all as dilute solutions in toluene.

commercial plastics (e.g., 1,4-bis(5-phenyloxazol-2-yl) benzene (POPOP)) are typically blue to violet emitters themselves, and their absorbance spectrums do not match well with the emission spectrum of DPA-DHS. In addition, the Stokes-shift for a wavelength shifter can not be excessively small (i.e., in an attempt to shorten the emission wavelength), as this would cause too much overlap of the shifters absorption/emission spectra, and would lead to excessive self absorption by the shifting dye, defeating its intended purpose. As such, the use of a green emitting wavelength shifter such as Coumarin 6 is necessary for the DPA-DHS/PVT system to maximize the number of out-coupled photons, although the primary consequence will be that the longer wavelength photons will have a lower detection efficiency by typical blue-sensitive PMTs (which in our measurements is factored out through spectral sensitivity correction as is described in section 2.4). Samples wavelength shifted with 0.05 wt.\% of Coumarin 6 were bright yellow-green in color, and representative photographs of the unshifted and shifted DPA-DHS samples along with EJ-299-34 for comparison are shown in fig. 6. 
The emission spectra of the commercial EJ-299-34, unshifted 50 wt.\% DPA-DHS/PVT,

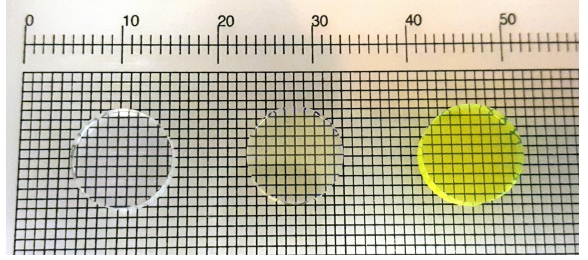

(a) Visibile Illumination

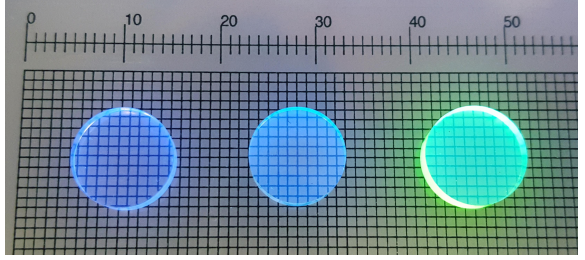

(b) UV Illumination

Figure 6: Photographs of (left) EJ-299-34, (middle) unshifted 50 wt.\% DPA-DHS/PVT, and (right) Coumarin-6 shifted 50 wt.\% DPA-DHS/PVT.

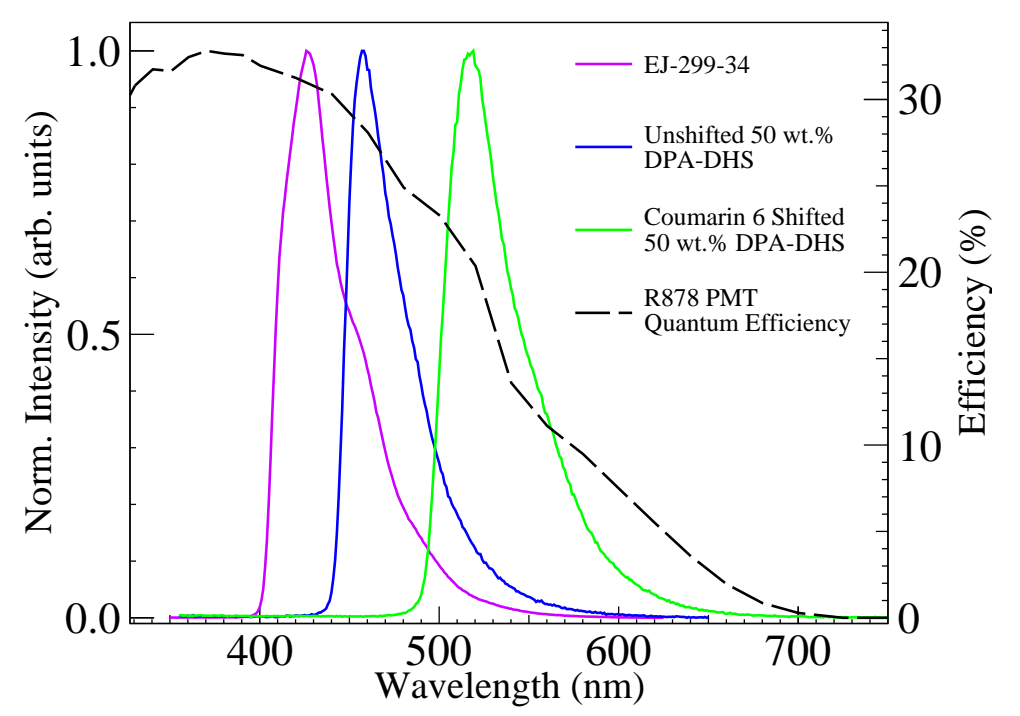

Figure 7: Emission Spectra of EJ-299-34, unshifted 50 wt.\% DPA-DHS/PVT, and Coumarin 6 shifted 50 wt.\% DPA-DHS/PVT.

and Coumarin 6 shifted 50 wt.\% DPA-DHS/PVT plastic scintillator samples is shown in fig. 7, along with a plot of the photo detection efficiency of a typical blue sensitive PMT photocathode (Hamamatsu R878). The emission for the unshifted samples was (1)

bright blue in color, with emission maxima centered around $450 \mathrm{~nm}$. This is slightly longer in wavelength than the commercial EJ-299-34 plastic, with an emission maximum centered at $425 \mathrm{~nm}$. Samples wavelength shifted with Coumarin 6 displayed 
bright green emissions with their maxima around $510 \mathrm{~nm}$.

\section{3. $\gamma$ Light Yields of DPA-DHS/PVT Samples}

Figure 8 plots the measured ${ }^{137} \mathrm{Cs}$ light yields as a function of DPA-DHS content for DPA-DHS/PVT samples with both no added wavelength shifter, and shifted with 0.05 wt.\% of Coumarin-6. Additionally, fig. 8 also plot the light yields for a series of

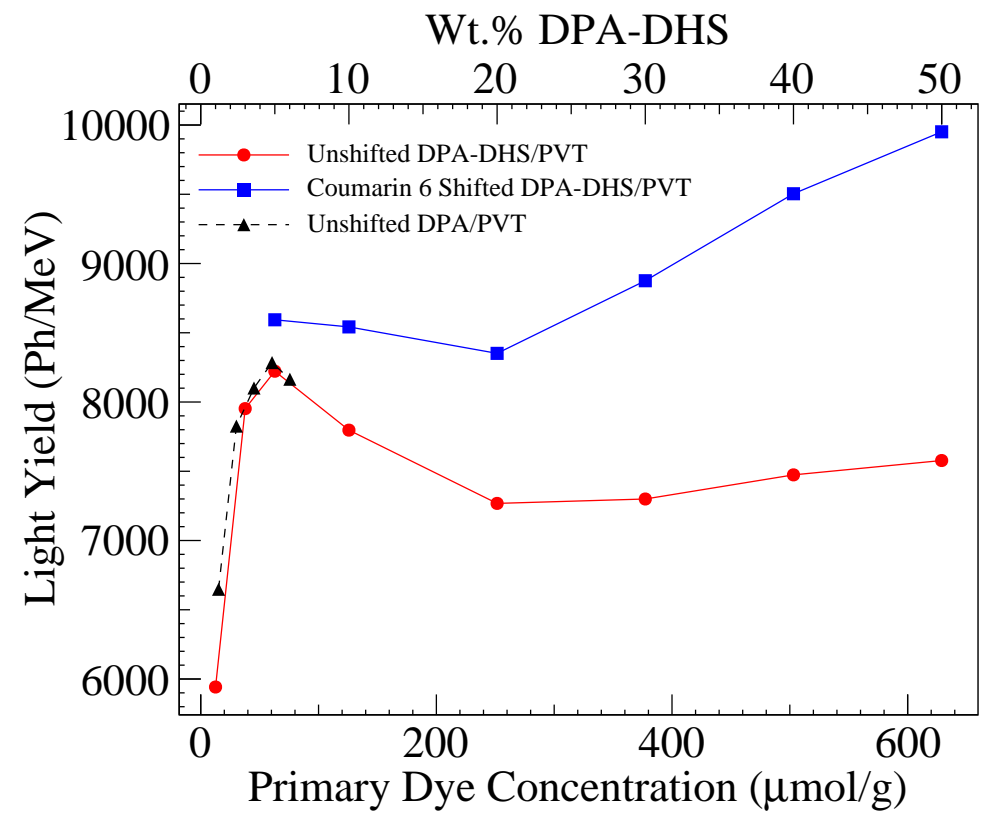

Figure 8: Light yields of unshifted DPA-DHS/PVT samples (top \& bottom axis), Coumarin 6 shifted DPADHS/PVT samples (top \& bottom axis), and unshifted DPA/PVT reference samples (bottom axis only).

comparative DPA/PVT samples with no added wavelength shifter, and prepared with DPA concentrations ranging from 0.5 to $2.5 \mathrm{wt} . \%$, which serve as an additional means of comparing the scintillation properties of the DPA-DHS monomer to that of unfunctionalized DPA. When the primary dye contents are compared on a concentration basis, it can clearly be seen that at least in the measurable range (for DPA) of lower dye loading, the light yields of the unshifted DPA/PVT and unshifted DPA-DHS/PVT are nearly identical to one another, with the DPA/PVT slightly outperforming the DPA-DHS/PVT samples. This slightly higher performance is in all likely hood directly attributable to 
the slightly higher $\phi_{P L}$ of DPA compared to DPA-DHS. The close correspondance of the light yields of DPA/PVT and DPA-DHS/PVT is a strong indicator that, at least in the lower concentration range, the scintillating properties of the two primary dyes are functionally equivalent to one another, and that copolymerization with the PVT matrix does not lead to quenching of the DPA-DHS dye. For both shifted and unshifted DPA-DHS/PVT, the samples with a loading of $20 \mathrm{wt} \%$ split the plots into two distinct regions. Below $20 \mathrm{wt} . \%$, the light yield in the unshifted samples is strongly rising up to a maximum of 8200 photons/MeV at $5 \mathrm{wt} . \%$, and then again falling down to a local minimum of 7300 photons/MeV at $20 \mathrm{wt} \%$. A similar behavior is also seen in the data for the shifted samples, although the light yield at $5 \mathrm{wt} \%$ loading is a slightly higher 8600 photons/MeV but falls much more slowly to a minimum of only 8350. Above 20 wt.\%, the light yields of the unshifted samples remain largely unchanged, except for a slight positive trend up to 7600 photons/MeV at $50 \mathrm{wt} \%$ loading, but for the shifted samples, a strong positive trend is evident up to 9950 photons/MeV, which is the highest for any of the samples DPA-DHS samples tested.

It is well known that DPA is relatively immune to the effects of concentration quenching, due to steric interaction of the phenyl rings at the 9,10-positions preventing the formation of excimer excited states [13, 25-28]. In addition, the $\gamma$ light yield for high quality single crystal DPA has been reported by van Loef et al. as 16,000 photons/ $\mathrm{MeV}$ [15]. If one assumes that these basic properties are approximately the same for the DPA cores in the DPA-DHS plastic, then the trends observed in our light yield data can be explained as follows. At a DPA-DHS content around $5 \mathrm{wt} \%$, the concentration is high enough to ensure efficient transfer of excitation energy from the PVT matrix (although this efficiency is quite likely less than unity), and yet the concentration is still low enough that effects of self absorption by DPA-DHS are still low enough that the overall light yield is maximized in this region. As the DPA-DHS content is increased up to $20 \mathrm{wt} . \%$, the efficiency of energy transfer from host to dye is relatively unchanged, but the increasing concentration of DPA-DHS is leading to a greater contribution of self absorption by DPA-DHS. For the unshifted samples, with no means of preventing loss due to self absorption, the light yield is falling in this region much faster than for the shifted ones. Above $20 \mathrm{wt} \%$, the same effects of an ever increas- 
ing contribution of DPA-DHS self absorption is still present, but at concentrations this high, it is no longer a valid assumption that primary excitation is occurring only on the PVT host, since at this point a significant volume fraction of the material is occupied by the DPA cores. Direct excitation of the cores by the passage of the high energy particle is expected to be a more efficient luminescent process, since no transfer of excitation energy needs to take place, and thus the luminescent efficiency for direct excitation depends only on the quantum yield of the dye. As the DPA-DHS content is increased up to $50 \mathrm{wt} \%$, a greater proportion of excitation is occurring directly on the DPA cores, and thus the overall scintillation efficiency (before factoring in the effects of self absorption) is increasing. For the unshifted samples, the increasing efficiency and the increasing effect of self absorption are directly competing to keep the light yield relatively unchanged, whereas for the shifted samples, the strong effect of the increasing efficiency is directly observable.

\section{4. $\gamma$ - and $\alpha$-Scintillation Decays of EJ-299-34 and DPA-DHS/PVT Samples}

Figure 9 compares the peak-normalized scintillation decay of the EJ-299-34 commercial plastic under both $\alpha$ - and $\gamma$-irradiation, while fig. 10 plots the corresponding data for both unshifted and Coumarin 6 shifted DPA-DHS samples at loadings of both $10 \mathrm{wt} . \%$ and $50 \mathrm{wt} . \%$ of primary dye. The decay curves obtained for EJ-299-34 typify the behavior expected for a material with good PSD performance, as there is a substantial increase in the fraction of light emitted as part of the decayed component upon going from $\gamma$ - to $\alpha$-excitation. While the decay curves of the unshifted and Coumarin 6 shifted 50 wt.\% DPA-DHS samples (fig. 10(b) and fig. 10(d)) do show increases in the delayed light component upon $\alpha$-excitation, the relative changes are noticeably less that that observed for EJ-299-34. The relative change in the fraction of delayed light is higher for the Coumarin 6 shifted sample as compared to the unshifted one. From the $\alpha$-decay data, it can be observed that at a time of $1 \mu \mathrm{s}$, the emission for all three of these samples has decayed to approximately the same value, nominally $0.1 \%$ of the peak value. However, the main difference in these samples lies in the proportion of delayed emission in the decay curves under $\gamma$-excitation. At $500 \mathrm{~ns}$, the emission from EJ-299-34 has decayed to $\sim 0.05 \%$ of the peak value, while at $1 \mu \mathrm{s}$, the emis- 


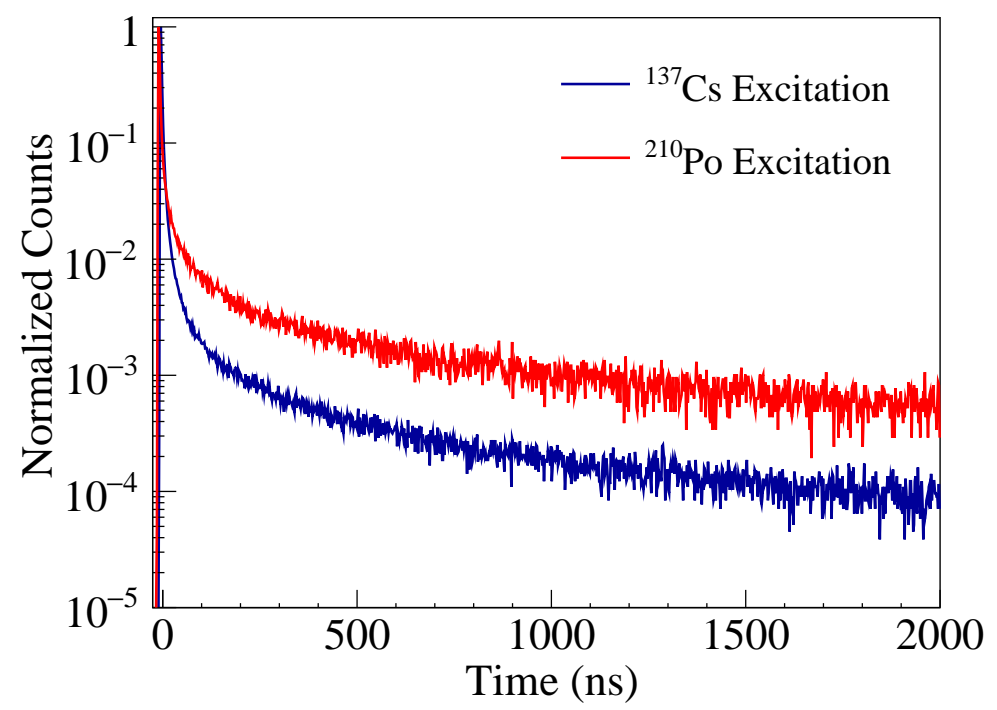

Figure 9: Scintillation Decay of EJ-299-34 under $\alpha$ - \& $\gamma$-irradiation.

sion has decayed to just above $0.01 \%$. For both the shifted and unshifted 50 wt. $\%$ DPA-DHS samples, the emission at these time points is noticeably higher, and for the unshifted sample in particular the emission at $500 \mathrm{~ns}$ has decayed to just under $0.1 \%$ of the peak value, and to $\sim 0.02 \%$ at $1 \mu \mathrm{s}$. This greater proportion of delayed emission under $\gamma$-excitation is the primary factor that is causing the decreased change in delayed emission observed for these samples. The decay curves of the unshifted and Coumarin 6 shifted 10 wt.\% DPA-DHS samples (fig. 10(a) and fig. 10(c), respectively) have significantly lower proportions of delayed light as compared to EJ-299-34 or the 50 wt.\% DPA-DHS samples. For instance, at a time of $1 \mu$ s, the emission under $\alpha$-excitation for both of these samples has decayed to a value just above $0.01 \%$ of the peak value. This is to be expected, since the primary dye content at this loading level is inadequate to allow for efficient triplet migration and subsequent annihilation. Furthermore, there is little difference in either sample between the fraction of delayed light between $\gamma$ - and $\alpha$-excitation.

The scintillation decay data also serves to provide additional evidence for the role that self-absorption plays in the difference in performance between the unshifted and 


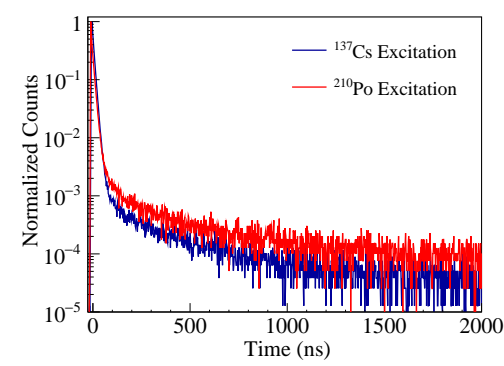

(a) $10 \mathrm{wt} . \%$ DPA-DHS (unshifted)

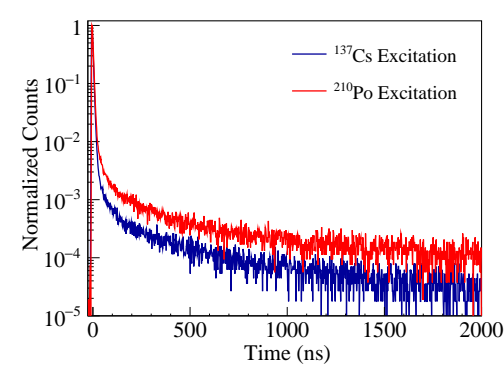

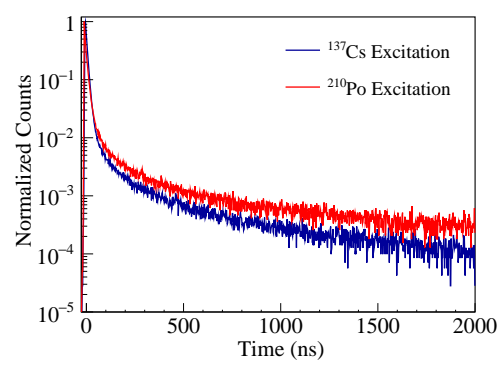

(b) 50 wt.\% DPA-DHS (unshifted)

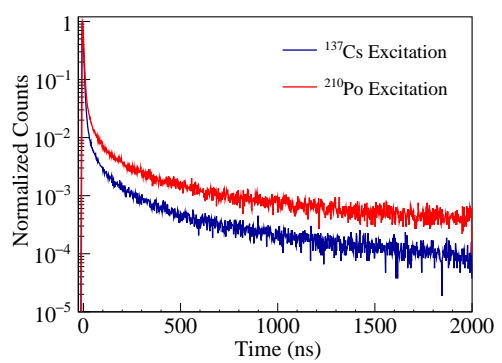

(c) $10 \mathrm{wt} . \%$ DPA-DHS (Coumarin 6 shifted) (d) $50 \mathrm{wt} \%$ DPA-DHS (Coumarin 6 shifted)

Figure 10: Comparison of Scintillation Decays of DPA-DHS/PVT Samples.

Coumarin 6 shifted DPA-DHS/PVT samples. Table 1 summarizes the values of the fast scintillation decay components of the tested samples that were obtained from fits of the lifetime data. From these values, it can clearly be seen that significantly longer prompt decay times were observed for both the $10 \mathrm{wt} . \%$ and $50 \mathrm{wt} . \%$ loadings of the unshifted samples ( $\sim 10 \mathrm{~ns})$ as compared to the shifted ( $\sim \mathrm{ns})$, despite the equivalent DPA-DHS content between the two groups. It is known that the observed lifetimes of organic scintillators depend both on the thickness and degree of self absorption with the material, since fluorescence photons absorbed by the sample may still be re-emitted, but the timing of this secondary emission is still governed by the molecular fluorescence decay lifetime [29]. Given this fact, it can be clearly seen that this increase in prompt decay time for the unshifted samples is directly attributable to their higher degree of self absorption. A further example of this can be seen in the Coumarin 6 shifted samples, where the $50 \mathrm{wt} . \%$ sample was found to have slightly longer prompt decay times than the $10 \mathrm{wt} . \%$ sample, which would suggest that at the highest DPA-DHS concentrations, 


\begin{tabular}{cccc}
\hline Sample & $\begin{array}{c}\text { DPA-DHS } \\
\text { Content }\end{array}$ & Source & $\begin{array}{c}\text { Fast Decay } \\
(\mathbf{n s})\end{array}$ \\
\hline EJ-299-34 & - & ${ }^{137} \mathrm{Cs}$ & 3.3 \\
& & ${ }^{210} \mathrm{Po}$ & 3.5 \\
\hline Unshifted & 10 wt.\% & ${ }^{137} \mathrm{Cs}$ & 9.9 \\
DPA-DHS & & ${ }^{210} \mathrm{Po}$ & 10.1 \\
& 50 wt.\% & ${ }^{137} \mathrm{Cs}$ & 9.6 \\
& & ${ }^{210} \mathrm{Po}$ & 10.5 \\
\hline Coumarin 6 & $10 \mathrm{wt} \%$ & ${ }^{137} \mathrm{Cs}$ & 4.7 \\
Shifted & & ${ }^{210} \mathrm{Po}$ & 5.0 \\
DPA-DHS & 50 wt.\% & ${ }^{137} \mathrm{Cs}$ & 5.4 \\
& & ${ }^{210} \mathrm{Po}$ & 6.2 \\
\hline
\end{tabular}

Table 1: Fast Scintillation Decay Components of Tested Samples.

the self absorption begins to directly compete with the much more efficient absorption by the wavelength shifting dye.

\section{5. $\alpha / \gamma$ PSD Properties of DPA-DHS/PVT Samples}

Figure 11 plots the 2D histograms of the PSD result $\left(\mathrm{Q}_{\text {tail }} / \mathrm{Q}_{\text {total }}\right)$ versus energy for data acquired for the commercial EJ-299-34 material, while figs. 12 and 13 plot the same results for unshifted DPA-DHS/PVT samples and samples shifted with 0.05 wt.\% Coumarin 6, respectively. The PSD figure of merit (FOM), defined as the separation between the two particle peaks over the sum of each respective FWHM, was measured as 2.43 using a $100 \mathrm{keV}$ threshold energy for the EJ-299-34 data. This value is well above the threshold criterion for useful PSD performance established by Zaitseva et al. as a value of 1.27 [6]. The data shown in fig. 11 also supplements the results reported by Nyibule et al. for the $\alpha / \gamma$ PSD properties of the original EJ-299-33 plastic formulation [30]. Figure 14 plots the FOM results for both unshifted and shifted DPA-DHS/PVT samples plotted as a function of primary dye content, using the same $100 \mathrm{keV}$ threshold energy. For the unshifted samples, while there is some increase in 


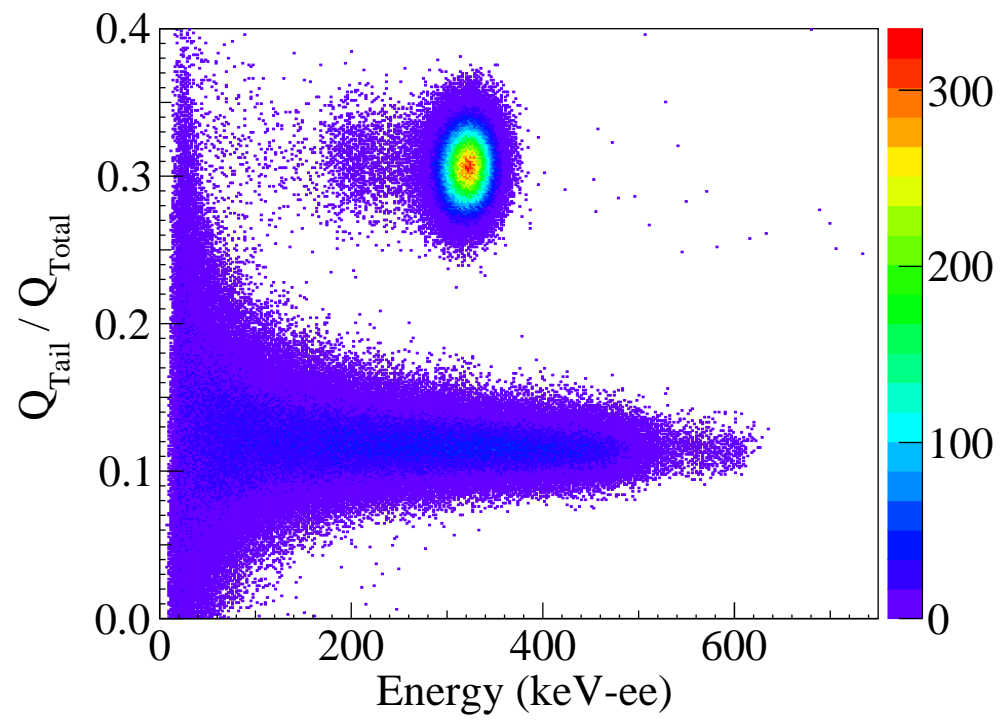

Figure 11: ${ }^{210} \mathrm{Po} /{ }^{137} \mathrm{Cs}$ PSD plot of commercial EJ-299-34 plastic.

the FOM as the content is increased above $10 \mathrm{wt} . \%$, the FOM trends toward a decrease above $20 \mathrm{wt} . \%$ and never is higher than 0.7 . In contrast, the wavelength shifted samples show an approximately linear trend towards increasing FOM values, with the $50 \mathrm{wt} . \%$ sample displaying the highest obtained FOM value of 1.05 , a value which is close to but less than the 1.27 threshold criterion. This is visually observable in fig. 13 that the $\alpha$ distribution is not cleanly separated from the $\gamma$ branch, with a slight degree of overlap between the two. The significantly poorer performance of the unshifted samples can, like the light yield results, be largely be attributed to the self absorption by the DPADHS dye, although some of the effect is also directly attributable to the differences in the decay curves shown in fig. 10. Since the charge comparison method relies on the measurement of relatively low light levels in the tail of the pulse, any decrease of detectable photons attributable to self absorption will have a significant and detrimental effect on the achievable PSD performance of the material. This is further evidenced by the large decrease in apparent $\alpha$ energy that is observed with increasing dye content. For the 10 wt.\% sample, the apparent energy of the alpha peak is less then that of EJ-299-34, with approximate values of $260 \mathrm{keV}$-ee and $320 \mathrm{keV}$-ee, respectively, and 


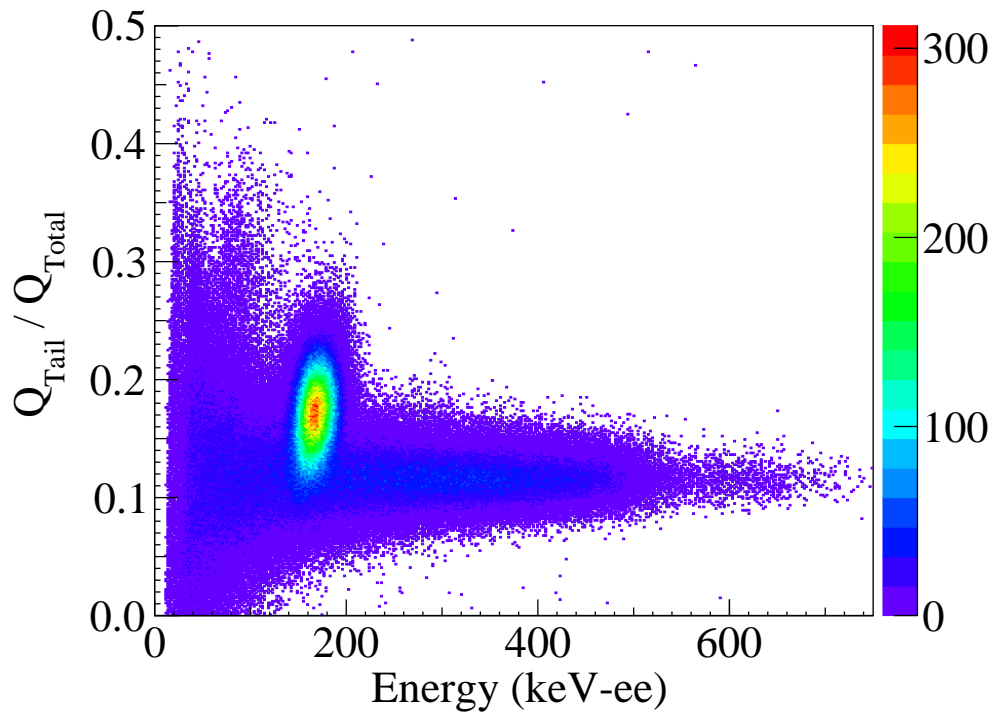

Figure 12: ${ }^{210} \mathrm{Po} /{ }^{137} \mathrm{Cs}$ PSD plot of unshifted $50 \mathrm{wt} . \%$ DPA-DHS/PVT.

decreases down to approximately $170 \mathrm{keV}$-ee for the $50 \mathrm{wt} \%$ sample. Since all of the energy of the incident $\alpha$-particles is absorbed within a thin surface layer on the face of the samples opposite of the PMT, the emitted light must travel the maximal distance through the samples and is thus will experience a large and uniform degree of loss due to self absorption, as compared to excitation across the entire volume of the sample in the case of incident $\gamma$-particles. On the contrary, the apparent alpha energy of the wavelength shifted samples is largely unchanged as the content of DPA-DHS is increased, decreasing slightly from $250 \mathrm{keV}$-ee to $235 \mathrm{keV}$ for the 10 and $50 \mathrm{wt} . \%$ samples, respectively.

There are potentially several factors that can contribute to the fact that the Coumarin 6 shifted samples do not match the performance level of the commercial EJ-299-34 plastic. From the photoluminescence spectra shown in fig. 7 , it is obvious from the longer wavelength emission of the Coumarin 6 samples that the overall detection efficiency by the R878 PMT for scintillation photons is significantly lower as compared to EJ-299-34. Thus while the light yields of EJ-299-24 and the shifted 50 wt.\% DPA-DHS are approximately the same, overall fewer photons are detected for the latter, which will 


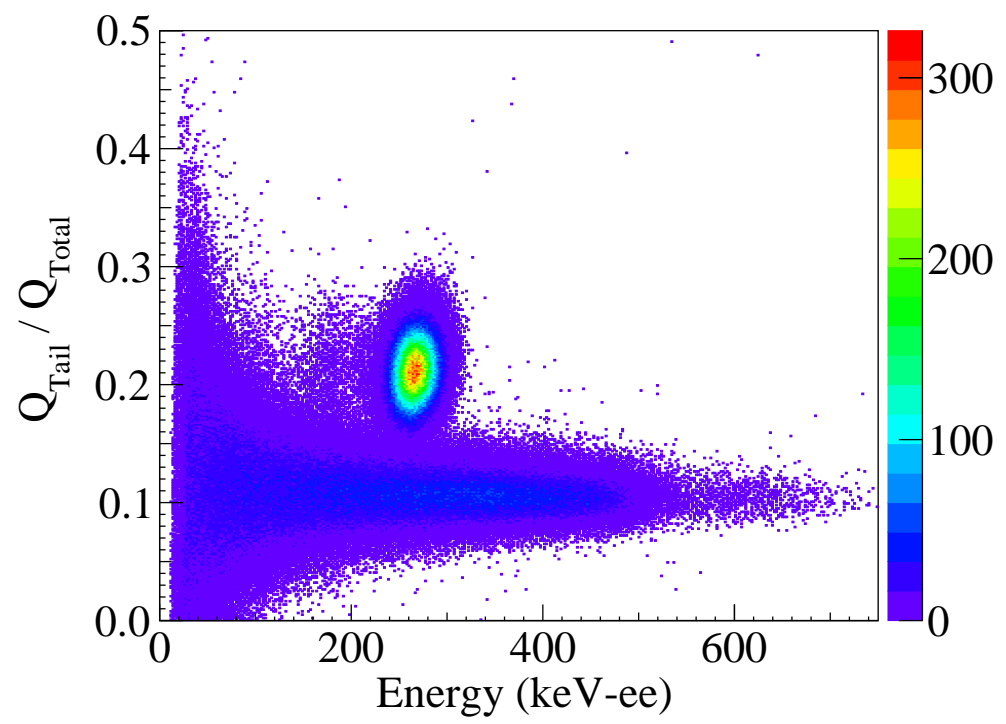

Figure 13: ${ }^{210} \mathrm{Po} /{ }^{137} \mathrm{Cs}$ PSD plot of Coumarin-6 shifted 50 wt.\% DPA-DHS/PVT.

result in loss of precision in terms of quantifying the relatively small amounts of delayed light emitted by the sample. However, given that the relative change in the slow component (going from $\gamma$ to $\alpha$ excitation) of the scintillation delay for the $50 \mathrm{wt} . \%$ DPA-DHS sample was considerably less than that for EJ-299-34, as is shown in fig. 9 and fig. 10, the relative PMT response alone is not enough to explain the poorer performance. As the emission of delayed fluorescence photons depends on the triplet-triplet annihilation process, which in turn depends on the diffusivity of triplet excitons within the material [31,32], it is possible that a significant difference in triplet diffusivity may be one of the factors responsible for the decreased PSD performance. Given that crystalline 9,10-diphenylanthracene is known to have good PSD performance [13-15], it is reasonable to presume that if a difference in triplet diffusivity is indeed responsible for the diminished performance, the structural modifications and/or polymer matrix of the DPA-DHS/PVT material are directly involved. Since the time scale for energy transfer from matrix to dye of triplet excitons should be much faster than the diffusion/annihilation process, non-radiative energy transfer to and from DPA cores should be the primary mechanism for triplet migration. In this sense, the polymer matrix, as 


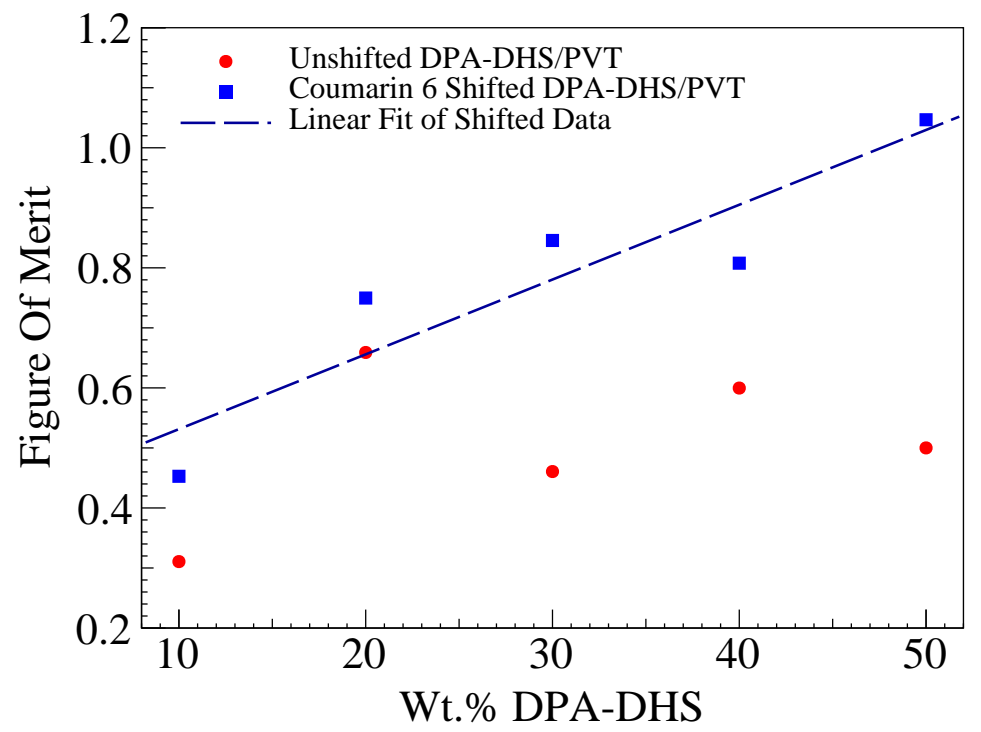

Figure 14: PSD figure of merits of both unshifted and Coumarin 6 wavelength shifted samples with DPADHS content from 10-50 wt.\%.

well as the flexible linking groups that bind the DPA cores will combine to increase the average DPA core spacing, which in general should have the effect of decreasing the efficiency and overall rate of energy transfer, leading to an overall decreased triplet diffusivity. If this hypothesis is correct, then it is expected that both a further increase in DPA core content, as well as a reduction in the linking group chain length could lead to a significant increase in PSD perfomance. However, both of these measures are problematic in terms of sample preparation; in particular, reduction of the linking group chain length will surely lead to both lower solubility and higher melting point of the dye monomer, making fabrication of such a plastic with even higher loadings even more problematic, if not impossible;

\section{Conclusion}

A novel, highly soluble, and polymerizable diphenylanthracene-based dye (DPADHS) was successfully synthesized, and plastic scintillator samples fabricated using this dye monomer were found to be hard, durable, and highly transparent. Dye concen- 
trations of at least 50 wt.\% when copolymerized with PVT were found to be readily achievable. Of all of the DPA-DHS/PVT compositions tested, the Coumarin 6 wavelength 50 wt.\% DPA-DHS was found to have the best performance, albeit notably less than the commercial EJ-299-34 plastic. While the unshifted DPA-DHS/PVT samples did show PSD properties, the strong effects of self-absorption led to significant loss of performance at the highest DPA-DHS concentrations. While the ultimate performance of the DPA-DHS/PVT material was just below the threshold limit for usability in real detectors, it does serve as a proof-of-concept for the possibility of highly loaded plastics stabilized via copolymerization, the lessons from which can be utilized in the design and synthesis of entirely new classes of dyes for PSD capable plastic scintillators. In doing so, it will be particularly important to focus on the design and synthesis of PSD-capable monomers that have shorter wavelength emission than the DPA-DHS monomer, so that they are compatible with traditional blue/violet emitting wavelength shifters, which is critical to achieving good spectral matching to typical PMT photocathodes.

\section{Acknowledgements}

The authors would like to graciously thank Dr. Nerine Cherapy and her team at Lawrence Livermore National Laboratory for additional tests they performed on our behalf that were not included as part of this work. In addition, the work reported herein was performed with the financial support of the Domestic Nuclear Detection Office (DNDO), part of the U.S. Department of Homeland Security (DHS), under the Grant ID 2014-DN-077-ARI071-0. The University of California Lab Fees Research Program (UCOP grant no. 12-LR-237678) is acknowledged for the initial exploratory investigation that lead to this work. This work is also based on the use of instruments at the UCLA Molecular Instrumentation Center (MIC) that are supported by the National Science Foundation under equipment grant no. CHE-1048804. 


\section{References}

[1] G. F. Knoll, Radiation Detection and Measurement, 4th Edition, John Wiley \& Sons, 2010.

[2] J. B. Birks, The Theory and Practice of Scintillation Counting, International series of monographs on electronics and instrumentation, v. 27, Pergamon Press, 1964.

[3] N. Zaitseva, A. Glenn, L. Carman, H. P. Martinez, R. Hatarik, H. Klapper, S. Payne, Scintillation properties of solution-grown trans-stilbene single crystals, Nuclear Instruments and Methods in Physics Research Section A: Accelerators, Spectrometers, Detectors and Associated Equipment 789 (2015) 8-15.

[4] F. D. Brooks, R. W. Pringle, B. L. Funt, Pulse shape discrimination in a plastic scintillator, Nuclear Science, IRE Transactions on 7 (2-3) (1960) 35-38.

[5] G. H. V. Bertrand, M. Hamel, S. Normand, F. Sguerra, Pulse shape discrimination between (fast or thermal) neutrons and gamma rays with plastic scintillators: state of the art, Nuclear Instruments and Methods in Physics Research Section A: Accelerators, Spectrometers, Detectors and Associated Equipment 776 (2015) $114-128$.

[6] N. Zaitseva, B. L. Rupert, I. Pawełczak, A. Glenn, H. P. Martinez, L. Carman, M. Faust, N. Cherepy, S. Payne, Plastic scintillators with efficient neutron/gamma pulse shape discrimination, Nuclear Instruments and Methods in Physics Research Section A: Accelerators, Spectrometers, Detectors and Associated Equipment 668 (2012) 88-93.

[7] N. Zaitseva, A. Glenn, L. Carman, R. Hatarik, S. Hamel, M. Faust, B. Schabes, N. Cherepy, S. Payne, Pulse shape discrimination in impure and mixed singlecrystal organic scintillators, IEEE Transactions on Nuclear Science 58 (6) (2011) $3411-3420$.

[8] E. T. Inc., Ej-299-34, http://www.eljentechnology.com/index.php/products/plasticscintillators/114-ej-299-33, online; accessed 11-19-2015. 
[9] H. P. Martinez, I. Pawelczak, A. M. Glenn, M. L. Carman, N. Zaitseva, S. Payne, Pulse shape discrimination in non-aromatic plastics, Nuclear Instruments and Methods in Physics Research Section A: Accelerators, Spectrometers, Detectors and Associated Equipment 771 (2015) 28-31.

[10] P. Blanc, M. Hamel, C. Dehé-Pittance, L. Rocha, R. B. Pansu, S. Normand, Neutron/gamma pulse shape discrimination in plastic scintillators: Preparation and characterization of various compositions, Nuclear Instruments and Methods in Physics Research Section A: Accelerators, Spectrometers, Detectors and Associated Equipment 750 (2014) 1-11.

[11] P. N. Zhmurin, V. N. Lebedev, V. D. Titskaya, A. F. Adadurov, D. A. Elyseev, V. N. Pereymak, Polystyrene-based scintillator with pulse-shape discrimination capability, Nuclear Instruments and Methods in Physics Research Section A: Accelerators, Spectrometers, Detectors and Associated Equipment 761 (2014) 9298.

[12] A. L. Vance, N. Mascarenhas, G. O’Bryan, S. Mrowka, Final ldrd report: advanced plastic scintillators for neutron detection., Tech. rep., Sandia National Laboratories (2010).

[13] Y. Scolnik, G. Shani, Z. B. Alfassi, Scintillation properties of crystalline 9,10diphenylanthracene: A proof for triplet-triplet annihilation, Nuclear Instruments and Methods in Physics Research Section A: Accelerators, Spectrometers, Detectors and Associated Equipment 254 (2) (1987) 389-392.

[14] E. V. van Loef, K. Markosyan, S. Mukhopadhyay, U. Shirwadkar, N. Zaitseva, S. Payne, K. S. Shah, Fast-neutron detection and pulse shape discrimination with diphenylanthracene and tetraphenylbutadiene, in: 2011 IEEE Nuclear Science Symposium Conference Record, 2011.

[15] E. V. van Loef, S. Mukhopadhyay, N. Zaitseva, S. Payne, K. S. Shah, Crystal growth and characterization of 9, 10-diphenylanthracene, Journal of Crystal Growth 352 (1) (2012) 103-105. 
[16] I. Berlman, Handbook of Fluorescence Spectra of Aromatic Molecules, 2nd Edition, Academic Press, 1971.

[17] N. V. Kornilov, V. A. Khriatchkov, M. Dunaev, A. B. Kagalenko, N. N. Semenova, V. G. Demenkov, A. J. M. Plompen, Neutron spectroscopy with fast waveform digitizer, Nuclear Instruments and Methods in Physics Research Section A: Accelerators, Spectrometers, Detectors and Associated Equipment 497 (2) (2003) 467-478.

[18] P. J. Sellin, G. Jaffar, S. D. Jastaniah, Performance of digital algorithms for $\mathrm{n} / \gamma$ pulse shape discrimination using a liquid scintillation detector, in: Nuclear Science Symposium Conference Record, 2003 IEEE, Vol. 2, IEEE, 2003, pp. 10571060.

[19] R. Aryaeinejad, J. K. Hartwell, D. F. Spencer, Comparison between digital and analog pulse shape discrimination techniques for neutron and gamma ray separation, in: Nuclear Science Symposium Conference Record, 2005 IEEE, Vol. 1, IEEE, 2005, pp. 500-504.

[20] Y. Kaschuck, B. Esposito, Neutron/ $\gamma$-ray digital pulse shape discrimination with organic scintillators, Nuclear Instruments and Methods in Physics Research Section A: Accelerators, Spectrometers, Detectors and Associated Equipment 551 (2) (2005) 420-428.

[21] M. Flaska, S. Pozzi, et al., Offline pulse-shape discrimination algorithms for neutron spectrum unfolding, in: Nuclear Science Symposium Conference Record, 2006. IEEE, Vol. 2, IEEE, 2006, pp. 752-758.

[22] J. Zhang, Z. C. Smith, S. W. Thomas III, Electronic effects of ring fusion and alkyne substitution on acene properties and reactivity, The Journal of organic chemistry 79 (21) (2014) 10081-10093.

[23] R. Young, P. Lovell, Introduction to Polymers, Third Edition, Polymer science, Taylor \& Francis, 2011. 
[24] P. Araújo, C. Sayer, R. Giudici, J. Poço, Techniques for reducing residual monomer content in polymers: a review, Polymer Engineering \& Science 42 (7) (2002) 1442-1468.

[25] E. J. Bowen, Fluorescence quenching in solution and in the vapour state, Transactions of the Faraday Society 50 (1954) 97-102.

[26] E. J. Bowen, D. W. Tanner, The photochemistry of anthracenes. part 3.interrelations between fluorescence quenching, dimerization, and photo-oxidation, Transactions of the Faraday Society 51 (1955) 475-481.

[27] J. B. Birks, J. B. Aladekomo, The photo-dimerization and excimer fluorescence of 9-methyl anthracene, Photochemistry and Photobiology 2 (4) (1963) 415-418.

[28] I. B. Berlman, Fluorescence of azulene and 9, 10-diphenylanthracene a reply to criticism, Chemical Physics Letters 21 (2) (1973) 344-345.

[29] J. B. Birks, W. A. Little, Photo-fluorescence decay times of organic phosphors, Proceedings of the Physical Society. Section A 66 (10) (1953) 921.

[30] S. Nyibule, E. Henry, W. Schröder, J. Tõke, L. Acosta, L. Auditore, G. Cardella, E. De Filippo, L. Francalanza, S. Gìani, et al., Radioluminescent characteristics of the ej 299-33 plastic scintillator, Nuclear Instruments and Methods in Physics Research Section A: Accelerators, Spectrometers, Detectors and Associated Equipment 728 (2013) 36-39.

[31] G. Laustriat, The luminescence decay of organic scintillators, Molecular Crystals 4 (1-4) (1968) 127-145.

[32] J. B. Birks, Energy transfer in organic systems vi. fluorescence response functions and scintillation pulse shapes, Journal of Physics B: Atomic and Molecular Physics 1 (5) (1968) 946. 